\title{
Four qubits can be entangled in nine different ways.
}

\author{
F.Verstraete ${ }^{1,2}$, J.Dehaene ${ }^{2}$, B.De Moor $^{2}$ and H.Verschelde ${ }^{1}$ \\ ${ }^{1}$ Ghent University, Department of Mathematical Physics and Astronomy Krijgslaan 281 (S9), B-9000 Gent, Belgium \\ ${ }^{2}$ Katholieke Universiteit Leuven, Department of Electrical Engineering, \\ Research Group SISTA Kasteelpark Arenberg 10, B-3001 Leuven, Belgium
}

\begin{abstract}
We consider a single copy of a pure four-partite state of qubits and investigate its behaviour under the action of stochastic local quantum operations assisted by classical communication (SLOCC). This leads to a complete classification of all different classes of pure states of four-qubits. It is shown that there exist nine families of states corresponding to nine different ways of entangling four qubits. The states in the generic family give rise to GHZ-like entanglement. The other ones contain essentially 2- or 3-qubit entanglement distributed among the four parties. The concept of concurrence and 3-tangle is generalized to the case of mixed states of 4 qubits, giving rise to a seven parameter family of entanglement monotones. Finally, the SLOCC operations maximizing all these entanglement monotones are derived, yielding the optimal single copy distillation protocol.
\end{abstract}

PACS numbers: 03.65.Ud

One of the open questions in the field of quantum information theory is to understand the different ways in which multipartite systems can be entangled. As the concept of entanglement is related to the non-local properties of a state, local quantum operations cannot affect the intrinsic nature of entanglement. It is therefore natural to define equivalence classes of states generated by the group of reversible SLOCC operations[1] 2]. In this letter we are concerned with SLOCC operations on one copy of a state, which means that we are considering actions under LOCC operations on one copy of a state without imposing that they can be achieved with unit certainty. Two states belonging to the same class are able to perform the same QIT-tasks, although with a different probability.

In the case of a single copy of an entangled pure state of two qubits, it is well known that it can be converted to the singlet state by SLOCC operations [3]. In the case of three entangled qubits, it was shown [2, 4, 5] that each state can be converted by SLOCC operations either to the GHZ-state $(|000\rangle+|111\rangle) / \sqrt{2}$, or to the Wstate $(|001\rangle+|010\rangle+|100\rangle) / \sqrt{3}$, leading to two inequivalent ways of entangling three qubits. The GHZ-state is generally considered as the state with the genuine 3partite entanglement, while the $\mathrm{W}$-state has the peculiar property of having the maximal expected amount of two-partite entanglement if one party is traced out [2]. In this letter we extend these results to the case of four qubits. Furthermore the widely celebrated entanglement measures concurrence [6] and 3-tangle [7], characterizing the amount of genuine two- and three-qubit entanglement, are generalized to the case of four qubits, giving rise to a 7 parameter family of entanglement monotones. The SLOCC filtering operations maximizing all these entanglement monotones are derived, and it is shown that these are the unique operations [8] (up to local unitaries) bringing a state into a locally stochastic form (i.e. bringing all local density operators equal to the identity). Fol- lowing Gisin [9], we claim that these operations maximize the true 4-partite entanglement.

Interestingly, we found that there exist eight families of pure 4-qubit states that cannot be brought into local stochastic form by finite SLOCC operations. These states do have the peculiar property that they have the maximal amount of 2- and/or 3-qubit entanglement shared between all 4 parties. In some sense their entanglement is maximally robust against the loss of one or two qubits.

An interesting feature about entanglement that emerges out of the results of this letter is the fact that a quantum state has only a finite susceptibility for entanglement. This will be illustrated by the fact that the operations maximizing the true 4-partite entanglement are precisely the operations that destroy all local correlations (i.e. the local density operators are made stochastic) and that also destroy the 3-partite entanglement (i.e. the 3tangle of the states obtained by tracing out one party becomes equal to zero). The states having maximal 2or 3-partite entanglement shared among the four parties on the other hand are exactly the states having zero genuine 4-partite entanglement (i.e. the 4-concurrences are all equal to zero).

Before developing the mathematical formalism, it should be noted that the study of states of four qubits is particularly interesting as the current experimental state of the art allows to entangle four photons [10, 11, 12] or ions 13]. Furthermore SLOCC operations can relatively easily be implemented on photons, and it is therefore of interest to implement the optimal SLOCC operations such as to yield a state with maximal 4-partite entanglement.

This letter is organized as follows. First we derive a simple way of determining whether two pure 4-qubit states are connected by local unitary operations. Next some advanced linear algebra is used to determine the orbits generated by SLOCC operations. This leads to 
nine different families of states, corresponding to nine essentially different ways of entangling four qubits, although only one family is generic. This analysis gives rise to seven independent entanglement monotones characterizing the 4-partite entanglement. Finally the optimal SLOCC operations are derived such as to maximize all these entanglement monotones.

Let us now first consider the problem to determine whether two pure 4-qubit states are equivalent up to local unitary operations. Therefore the following accident in Lie-group theory can be exploited:

$$
S U(2) \otimes S U(2) \simeq S O(4)
$$

Here $S O(4)$ denotes the family of real orthogonal matrices with determinant equal to 1 . More specifically, it holds that $\forall U_{1}, U_{2} \in S U(2): T\left(U_{1} \otimes U_{2}\right) T^{\dagger} \in S O(4)$ where

$$
T=\frac{1}{\sqrt{2}}\left(\begin{array}{cccc}
1 & \cdot & \cdot & 1 \\
\cdot & i & i & \cdot \\
\cdot & -1 & 1 & \cdot \\
i & \cdot & \cdot & -i
\end{array}\right)
$$

A pure state of four qubits is parameterized by a four index tensor $\psi_{i_{i} i_{2} i_{3} i_{4}}$ with $i_{j} \in\{1,2\}$. This tensor can be rewritten as a $4 \times 4$ matrix $\tilde{\psi}$ by concatenating the indices $\left(i_{1}, i_{2}\right)$ and $\left(i_{3}, i_{4}\right)$. Next we define the matrix $\mathrm{R}$ as

$$
R=T \tilde{\psi} T^{\dagger}
$$

It is then straightforward to show that a local unitary transformation $\left|\psi^{\prime}\right\rangle=U_{1} \otimes U_{2} \otimes U_{3} \otimes U_{4}|\psi\rangle$ results in a transformation $R^{\prime}=O_{1} R O_{2}$ with $O_{1}, O_{2} \in S O(4)$ and $O_{1}=T\left(U_{1} \otimes U_{2}\right) T^{\dagger}, O_{2}=T\left(U_{3} \otimes U_{4}\right)^{T} T^{\dagger}$. A normal form under local unitary operations can now be imposed as follows: make the $(1,1)$ entry of $R$ real by multiplying the whole matrix with the appropriate phase, and use $O_{1}$ and $O_{2}$ to diagonalize the real part of $R$ through the unique real singular value decomposition. This procedure eliminates all 13 degrees of freedom of the local unitary operations, and two states are therefore equivalent up to local unitary operations iff they have the same normal form.

Next we move to the central problem of this letter, namely characterizing the local orbits generated by SLOCC operations of the form

$$
\left|\psi^{\prime}\right\rangle=A_{1} \otimes A_{2} \otimes A_{3} \otimes A_{4}|\psi\rangle
$$

with $\left\{A_{i}\right\}$ full rank and therefore invertible $2 \times 2$ matrices. There is no restriction in choosing $\left\{A_{i}\right\} \in S L(2, \mathbb{C})$, and then a new useful accident arises:

$$
S L(2, \mathbb{C}) \otimes S L(2, \mathbb{C}) \simeq S O(4, \mathbb{C}) .
$$

$S O(4, \mathbb{C})$ denotes the non-compact group of complex orthogonal matrices $O^{T} O=I_{4}$. Again it holds that $\forall A, B \in S L(2, \mathbb{C}): T\left(A_{1} \otimes A_{2}\right) T^{\dagger} \in S O(4, \mathbb{C})$ with $T$ given in equation (1), and SLOCC operations therefore correspond to left and right multiplication of $R(2)$ with complex orthogonal matrices. The challenge is now to exploit the two times 12 degrees of freedom of these complex orthogonal matrices to bring $A$ into an unique normal form with maximal 8 real degrees of freedom left. This will be possible using some advanced techniques of linear algebra.

We will now state a technical theorem that is a generalization of the singular value decomposition to complex orthogonal matrices.

Theorem 1 Given a complex $n \times n$ matrix $R$, then there always exist complex square orthogonal matrices $O_{1}$ and $O_{2}$ such that $R^{\prime}=O_{1} R O_{2}$ is a unique direct sum of blocks of the form:

1. $m \times m$ blocks of the form $\left(\lambda_{j} I_{m}+S_{m}\right)$ being symmetric Jordan blocks (see for example [1] 4.4.9), and $\lambda_{j}$ is a complex parameter (note that the case $m=1$ corresponds to the scalar case).

2. $m \times m$ blocks consisting of an upper left $\left(m_{1}+1\right) \times m_{1}$ part being the matrix obtained by taking the odd rows and even columns of an $\left(2 m_{1}+1\right) \times\left(2 m_{1}+1\right)$ symmetric Jordan block, and a lower right $\left(m-m_{1}-1\right) \times\left(m-m_{1}\right)$ part being the transpose of the matrix obtained by taking the odd rows and even columns of a $\left(2\left(m-m_{1}\right)-1\right) \times$ $\left(2\left(m-m_{1}\right)-1\right)$ symmetric Jordan block.

Proof: Consider the $2 n \times 2 n$ complex symmetric matrix

$$
P=\left(\begin{array}{cc}
0 & R \\
R^{T} & 0
\end{array}\right) .
$$

Due to theorem 5 in ch.XI of 15, there exists a complex orthogonal $Q$ such that $P=Q P^{\prime} Q^{T}$ with $P^{\prime}$ a direct sum of symmetric $m \times m$ Jordan blocks $J_{i}$ with eigenvalue $\lambda_{i}$. Next we observe that whenever $\left[v_{1} ; v_{2}\right]\left(v_{1}\right.$ and $v_{2}$ both have $n$ rows such that $\left[v_{1} ; v_{2}\right]$ has $2 n$ rows) is the eigenspace of $P$ corresponding to a symmetric Jordan block $J_{i}$, then $\left[v_{1} ;-v_{2}\right]$ is the eigenspace of $P$ corresponding to a Jordan block $-J_{i}$. Due to the uniqueness of the Jordan canonical decomposition, these eigenspaces will be either linearly independent (this holds for example for sure if the corresponding eigenvalue is different from zero), or equal to each other (which implies that the corresponding eigenvalue is equal to zero). If the first case applies, both $v_{1}$ and $v_{2}$ are orthogonal matrices.

The second degenerated case however is more difficult. In this case, it holds that $\left[v_{1} ; v_{2}\right]=\left[v_{1} ;-v_{2}\right] Q$ for some orthogonal $Q$. Let us first calculate the standard nonsymmetric Jordan canonical form $\tilde{J}$ of the symmetric Jordan block with eigenvalue $0: J=U^{\dagger} \tilde{J} U$ with $U$ unitary and symmetric. If we define $\left[x_{1} ; x_{2}\right]=\left[v_{1} ; v_{2}\right] U^{\dagger}$ and $\tilde{Q}=U^{\dagger} Q U$, the following identities hold: $\tilde{Q}^{T} \operatorname{Sip} \tilde{Q}=\operatorname{Sip}$, $\tilde{Q} \tilde{J}=-\tilde{J} \tilde{Q}$ and $\left[x_{1} ; x_{2}\right]^{T}\left[x_{1} ; x_{2}\right]=\operatorname{Sip}($ the matrix Sip is defined as the matrix permuting all vectors $\left[x_{1}, x_{2} \cdots x_{n}\right]$ to $\left.\left[x_{n}, x_{n-1} \cdots x_{1}\right]\right)$. The conditions on $\tilde{Q}$ imply that $\tilde{Q}$ 
is equal to the matrix $\tilde{Q}_{i j}= \pm(-1)^{i} \delta_{i j}$. Therefore $\left[x_{1} ; x_{2}\right]$ is either of the form

$$
\left(\begin{array}{l}
x_{1} \\
x_{2}
\end{array}\right)=\left(\begin{array}{cccccc}
a_{1} & 0 & b_{1} & 0 & c_{1} & \cdots \\
0 & a_{2} & 0 & b_{2} & 0 & \cdots
\end{array}\right)
$$

or

$$
\left(\begin{array}{l}
x_{1} \\
x_{2}
\end{array}\right)=\left(\begin{array}{cccccc}
0 & a_{1} & 0 & b_{1} & 0 & \cdots \\
a_{2} & 0 & b_{2} & 0 & c_{2} & \cdots
\end{array}\right) .
$$

Due to the constraint $\left[x_{1} ; x_{2}\right]^{T}\left[x_{1} ; x_{2}\right]=\mathrm{Sip}$, the row dimension of $\left[x_{1} ; x_{2}\right]$ and therefore of $J$ has to be odd, as otherwise the upper rightmost entry cannot be equal to 1 . Retransforming to the original picture with the unitary $U$, it holds that this structure is preserved, and the eigenspace $\left[v_{1} ; v_{2}\right]$ is of a form (6) or (7).

As the dimension of a $J_{i}$ giving rise to the degenerated case has to be odd, it is compulsory that there is an even number of degenerated cases (indeed, the non-degenerate cases give rise to two times a similar block and the total dimension is even). More precisely, for each $\left[v_{1} ; v_{2}\right]_{j}$ of the form (6), there has to exist a $\left[v_{1} ; v_{2}\right]_{k}$ of the form (7) (eventually of different dimension). The eigenstructure of such pairs of degenerate cases can then be brought into the form

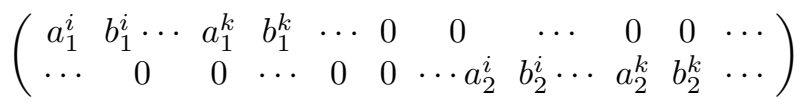

by right multiplication with a permutation matrix $W$. The effect on $J_{i}$ and $J_{k}$ is to transform them as

$$
W^{T}\left(\begin{array}{cc}
J_{i} & 0 \\
0 & J_{k}
\end{array}\right) W=\left(\begin{array}{cccc}
0 & 0 & K_{i} & 0 \\
0 & 0 & 0 & K_{k}^{T} \\
K_{i}^{T} & 0 & 0 & 0 \\
0 & K_{k} & 0 & 0
\end{array}\right)
$$

where $K_{\nu}$ represents the matrix obtained by taking the odd rows and even columns of the symmetric Jordan block $J_{\nu}$.

Collecting all the pieces, it is now easily verified that the canonical form obtained is exactly of the form stated in the theorem. This completes the proof.

Due to the equivalence of $S L(2, \mathbb{C}) \otimes S L(2, \mathbb{C})$ and $S O(4, \mathbb{C})$, the normal forms arising in the above lemma will immediately yield a natural representative state for each class of 4-qubit states connected by SLOCC operations. The normal form encodes the genuine nonlocal properties of the state, while the SLOCC operators needed to bring the state into normal form characterize the local information. The following classification is obtained:

Theorem 2 A pure state of 4 qubits can, up to permutations of the qubits, be transformed into one of the following 9 families of states by determinant 1 SLOCC operations (3):

$$
G_{a b c d}=\frac{a+d}{2}(|0000\rangle+|1111\rangle)+\frac{a-d}{2}(|0011\rangle+|1100\rangle)
$$

$$
\begin{aligned}
& +\frac{b+c}{2}(|0101\rangle+|1010\rangle)+\frac{b-c}{2}(|0110\rangle+|1001\rangle) \\
& L_{a b c_{2}}=\frac{a+b}{2}(|0000\rangle+|1111\rangle)+\frac{a-b}{2}(|0011\rangle+|1100\rangle) \\
& +c(|0101\rangle+|1010\rangle)+|0110\rangle \\
& L_{a_{2} b_{2}}=a(|0000\rangle+|1111\rangle)+b(|0101\rangle+|1010\rangle) \\
& +|0110\rangle+|0011\rangle \\
& L_{a b_{3}}=a(|0000\rangle+|1111\rangle)+\frac{a+b}{2}(|0101\rangle+|1010\rangle) \\
& +\frac{a-b}{2}(|0110\rangle+|1001\rangle) \\
& +\frac{i}{\sqrt{2}}(|0001\rangle+|0010\rangle+|0111\rangle+|1011\rangle) \\
& L_{a_{4}}=a(|0000\rangle+|0101\rangle+|1010\rangle+|1111\rangle) \\
& +(i|0001\rangle+|0110\rangle-i|1011\rangle) \\
& L_{a_{2} 0_{3 \oplus \overline{1}}}=a(|0000\rangle+|1111\rangle)+(|0011\rangle+|0101\rangle+|0110\rangle) \\
& L_{0_{5 \oplus \overline{3}}}=|0000\rangle+|0101\rangle+|1000\rangle+|1110\rangle \\
& L_{0_{7 \oplus \overline{1}}}=|0000\rangle+|1011\rangle+|1101\rangle+|1110\rangle
\end{aligned}
$$

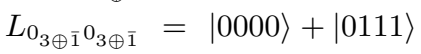

The complex parameters $a, b, c, d$ are the unique eigenvalues of $P$ (5) with non-negative real part, and the indices $L_{\alpha \beta} \ldots$ are representative for the Jordan block structure of $P$ (e.g. $L_{a_{2} 0_{3 \oplus \overline{1}}}$ means that the eigenstructure of $P$ consists of two $2 \times 2$ Jordan blocks with eigenvalues a and $-a$, and a degenerated pair of dimension respectively 3 and 1).

Proof: If theorem 1 is applied to a $4 \times 4 R$, it is easily checked that 12 different families arise where a family is defined as having Jordan and degenerated Jordan blocks of specific dimension. Note however that the orthogonal matrices obtained by application of the theorem can have determinant equal to -1 , while the SLOCC operations correspond to an orthogonal matrix with determinant +1 ; this is however not a problem as these operations correspond to SLOCC operations followed by a permutation of the qubits $(1 \leftrightarrow 2)$ or $(3 \leftrightarrow 4)$. One can proceed by checking that permutations of qubits $(2 \leftrightarrow 3)$ or $(1 \leftrightarrow 4)$ transform different families into each other. It is indeed true that $R=J_{1}(a) \oplus J_{1}(b) \oplus K_{3 \oplus \overline{1}}$ transforms into $R^{\prime}=J_{2}(a) \oplus J_{2}(b)$ if qubit 2 and 3 are permuted. This also happens in the case $J_{1}(a) \oplus K_{5 \oplus \overline{1}} \rightarrow J_{4}(a)$. Moreover it can be shown that $J_{1}(a) \oplus K_{3 \oplus \overline{3}}$ is equivalent to $J_{1}(a) \oplus J_{3}(0)$. Therefore only 9 essentially different normal forms are retained.

A generic pure state of 4 qubits can always be transformed to the $G_{a b c d}$ state. This state is peculiar in the sense that all local density operators, obtained by tracing out all parties but one, are proportional to the identity. As shown in [8], this is the unique state (up to local unitary operations) with this property of all states connected by SLOCC operations. In the light of the results of Gisin [9] and Nielsen about majorization 16, 17, we claim that this is the state with maximal 4-partite entanglement on the complete orbit generated by SLOCC 
operations: the more entanglement, the more local entropy. In a later section this argument will be made hard by showing that a whole class of entanglement monotones are indeed maximized for the locally stochastic state.

It is interesting to note that the 3 -tangle [7] of the mixed states obtained by tracing out one party of this $G_{a b c d}$ state is always equal to zero. Indeed, if the rightunitary matrix $\mathrm{U}$

$$
\begin{aligned}
U & =\frac{1}{\sqrt{2\left(1+|\beta|^{2}\right)}}\left(\begin{array}{cccc}
1 & \beta & 1 & -\beta \\
\beta & 1 & -\beta & 1
\end{array}\right) \\
\beta & =\sqrt{-q+\sqrt{q^{2}-r}} \\
q & =8 a^{2} d^{2}+8 b^{2} c^{2}-4 a^{2} b^{2}-4 a^{2} c^{2}-4 d^{2} b^{2}-4 d^{2} c^{2} \\
r & =\left(a^{2}-d^{2}\right)\left(b^{2}-c^{2}\right)
\end{aligned}
$$

is applied to the $8 \times 2$ matrix

$$
\left(\begin{array}{cccccccc}
a+d & \cdot & \cdot & a-d & \cdot & b+c & b-c & \cdot \\
\cdot & b-c & b+c & \cdot & a-d & \cdot & \cdot & a+d
\end{array}\right)^{T}
$$

being the square root of the density operator obtained by tracing out the first qubit, 4 3-qubit W-states are obtained. If we define the mixed 3-tangle [8] as the convex roof of the square root of the 3-tangle, this quantity is clearly equal to zero. Therefore the SLOCC operations maximizing the 4-partite entanglement result in a loss of all true 3-partite entanglement. This is reminiscent to the case of 3 qubits where the 2-qubit state obtained by tracing out one particle of a GHZ-state is separable.

Let us next discuss some specific examples. A completely separable state belongs to the family $L_{a b c_{2}}$ with $a=b=c=0$. If only two qubits are entangled, an EPR state arises belonging to the family $L_{a_{2} b_{2}}$ with $a=b=0$. A state consisting of two EPR-pairs belongs to $G_{a b c d}$ with $(a=1 ; b=c=d=0)$ or $a=b=c=d$, depending

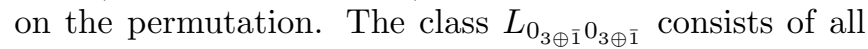
3-qubit GHZ states accompanied with a separable qubit, while the 3 -qubit W-state belongs to the family $L_{a_{2} 0_{3 \oplus \overline{1}}}$ with $a=0$.

The 4-qubit $\mid \Phi_{4}$-state $18 \mid$ belongs to the generic family, while the 4-qubit W-state $(|0001\rangle+|0010\rangle+|0100\rangle+$ $|1000\rangle) / 2$ belongs to the family $L_{a b_{3}}$ with $a=b=0$. This W-state can be shown to have a mixed 3 -tangle equal to zero, but has a concurrence of $1 / 2$ when whatever two qubits are traced out. On the contrary the state $L_{O_{7 \oplus \overline{1}}}$ has all concurrences equal to zero if two qubits are traced out. This state is completely symmetric in the permutation of the qubits 2,3 and 4 . It has the property of having a mixed 3 -tangle equal to $1 / 2$ if particle 2,3 or 4 is traced out. This can be proven by considering the $8 \times 2$ "square root"

$$
\frac{1}{2}\left(\begin{array}{llllllll}
1 & \cdot & \cdot & \cdot & \cdot & \cdot & \cdot & 1 \\
\cdot & \cdot & \cdot & 1 & \cdot & \cdot & 1 & \cdot
\end{array}\right)^{T}
$$

Some straightforward calculations show that the average square root of the 3-tangle of the vectors obtained by multiplying this matrix with whatever $2 \times n$ right-unitary matrix is equal to $1 / 2$. Similar arguments show that only three-qubit $\mathrm{W}$-type entanglement $(\tau=0)$ is retained if the first qubit is traced out.
The state $L_{0_{5 \oplus \overline{3}}}$ is somehow a hybrid of both the 4qubit W-state and $L_{O_{7 \oplus \overline{1}}}$. Again a mixed 3-tangle of $1 / 2$ is obtained if qubit 2,3 or 4 is traced out, a mixed 3-tangle equal to zero if qubit 1 is traced out, but now the mixed state obtained by tracing out qubit 1 and ( 3 or 4 ) has a concurrence equal to $1 / 2$, while the other concurrences vanish.

Another interesting state belongs to the family $L_{a_{4}}$ with $a=0$ : $|\psi\rangle=(|0001\rangle+|0110\rangle+|1000\rangle) / \sqrt{3}$. Its mixed 3 -tangle equals $2 / 3$ in the case of tracing out qubit 1 or 4 and vanishes otherwise. Moreover the concurrence vanishes everywhere if 2 qubits are traced out except in the case of tracing out qubit 2 and 3 , resulting in a concurrence of $2 / 3$.

After this zoological survey, let us next move on to the topic of entanglement monotones. The complex eigenvalues of $\mathrm{P}(5)$, given by $\pm(a, b, c, d)$, are the only invariants under all determinant 1 SLOCC operations (note that an eigenvalue 0 is associated to the degenerated Jordan blocks). In \& it was proven that all real positive functions of the parameters of a pure state that are linearly homogeneous in $\rho$ and remain invariant under determinant 1 SLOCC operations, are entanglement monotones (in the case of mixed states they are defined by the convex roof formalism). Therefore all real positive homogeneous functions of $\left(a^{2}, b^{2}, c^{2}, d^{2}\right)$ are entanglement monotones, such as

$$
M_{\alpha}(\psi)=\left|a^{\alpha}+b^{\alpha}+c^{\alpha}+d^{\alpha}\right|^{2 / \alpha} .
$$

Taking into account one degree of freedom due to the phase, this gives rise to a seven-parameter family of entanglement monotones. All these entanglement monotones are maximized by the operations making the density matrix locally stochastic [8] (meaning that the identity is obtained when all qubits but one are traced out). The optimal single-copy distillation procedure for a generic pure state is therefore to implement the SLOCC operations bringing it into its normal form $G_{a b c d}$. This is in complete accordance with the results of Nielsen on majorization [16]. Note that all the other normal forms can only be brought into the local stochastic normal form by a filtering procedure whose probability of success tends to zero [8].

In summary, we have identified all different families of pure states of 4 qubits generated by SLOCC operations. Only one family is generic, and all states in it can be made locally stochastic by SLOCC operations. The same SLOCC operations represent the optimal single-copy distillation protocol. The eight other families correspond to states having some kind of degenerated 4-partite entanglement and are the 4-partite generalizations of the 3-partite W-state. 
246:1, 1998.

[10] S. Gasparoni G. Weihs A. Zeilinger. J. Pan, M. Daniell. Phys. Rev. Lett., 86:4435, 2001.

[1] C.H. Bennett, S. Popescu, D. Rohrlich, J. A. Smolin, and

[11] Harald Weinfurter and Marek Zukowski. quant$\mathrm{ph} / 0103049$.

[2] W. Dur, G. Vidal, and J. 1. Cirac. Phys. Rev. A, 62:062314, 2000.

[3] H.K. Lo and S. Popescu. quant-ph/9707038.

[4] A. Acin, E. Jane, W. Dür, and G. Vidal. Phys. Rev. Lett., 85:4811, 2000.

[5] F. Verstraete, J. Dehaene, and B. De Moor. Phys. Rev. A, 65:032308 (2002).

[6] W. Wootters. Phys. Rev. Lett., 80:2245, 1998.

[7] V. Coffman, J. Kundu, and W.K. Wootters. Phys. Rev. A, 61:052306, 2000.

[8] F. Verstraete, J. Dehaene, and B. De Moor. quant$\mathrm{ph} / 0105090$.

[9] N. Gisin and H. Bechmann-Pasquinucci. Phys. Lett. A,

[12] A. Lamas-Linares, J. C. Howell, and D. Bouwmeester. Nature, 412:887, 2001.

[13] C.A. Sackett et al. Nature, 404:256, 2000.

[14] R. Horn and C. Johnson. Matrix Analysis. Cambridge University Press, 1985.

[15] F.R. Gantmacher. The theory of matrices. Chelsea Publishing Company, New York, 1959.

[16] M. Nielsen. Phys. Rev. Lett., 83:436, 1999.

[17] M. Nielsen and J. Kempe. Phys. Rev. Lett., 86:5184, 2001.

[18] H.J. Briegel and R. Raussendorf. Phys. Rev. Lett., 86:910, 2001. 